\title{
DEL PRINCIPIO QUIEN CONTAMINA PAGA AL PRINCIPIO QUIEN SE BENEFICIA PAGA: NUEVOS INSTRUMENTOS ECONÓMICOS EN MATERIA AMBIENTAL
}

Tania García López

Doctorado en Derecho ambiental por la Universidad de Alcalá de Henares, España. Investigadora en Universidad Veracruzana Investigadora Nacional nivel 2, CONACYT, México E-mail: Tgar70@gmail.com

\begin{abstract}
RESUMEN
El uso de instrumentos económicos al servicio de las políticas públicas ambientales se ha ido generalizando en todos los países especialmente durante los últimos 10 años. Existen un buen número de técnicas y aproximaciones a este tipo de instrumentos. En este trabajo ofrecemos un estudio que se centra en los principios que los rigen y hacemos énfasis en los instrumentos económicos más recientes como es el pago por servicios ambientales, el intercambio de deuda por naturaleza o los bonos de conservación.
\end{abstract}

Palabras-clave: Instrumentos económicos; quien contamina paga; quien se beneficia paga 


\title{
FROM POLLUTER PAYS PRINCIPLE TO BENEFI- CIARY PAYS PRINCIPLE: NEW ECONOMIC INSTRU- MENTS FOR ENVIRONMENTAL PROTECTION
}

\begin{abstract}
Economic instruments for environmental protection have been increasing, specially during last ten years and its use has been growing all around the countries. There are a lot of techniques and approaches to these kind of instruments. This study aims to offer a look focused on the principles which serve as guide for these economic instruments. The work also underline the most recently developed economic instruments, like paying for ecosystem services, exhanging debt by nature or conservation bonds.
\end{abstract}

Keywords: Economic instruments; polluter pays principle; beneficiary pays principle 


\section{INTRODUCCIÓN}

El desarrollo de instrumentos económicos al servicio de las políticas públicas ambientales se ha ido generalizando en todos los países especialmente durante los últimos 10 años.

Estos instrumentos económicos, que complementan a los denominados de "comando y control" o instrumentos de control directo de la contaminación, encuentran sus bases jurídicas en muy diferentes sectores de los ordenamientos jurídicos nacionales, siendo su desarrollo de lo más heterogéneo, así como su diseño y aplicación.

Además de lo anterior, es necesario tomar en cuenta que en la actualidad conviven instrumentos económicos tradicionales adaptados a fines ambientales, como es el caso de algunos seguros o de muchos de los impuestos ambientales y otros que surgen, ya, desde una óptica estrictamente ambiental, como es el caso de los certificados de emisión negociables, por citar sólo algún ejemplo.

Al mismo tiempo se han ido creando instrumentos económicos nuevos, que encuentran un difícil acomodo dentro de los sistemas legalesexistentes y que se basan en principios de derecho ambiental que han sufrido, a lo largo de las últimas décadas, cambios importantes en cuanto a su alcance y significado.

Asimismo, existen instrumentos económicos de carácter obligatorio, como es el caso de los impuestos y otros de carácter voluntario, como son algunos fondos ambientales que surgen del sector privado.

Por otra parte, y aunque en todos los casos los instrumentos económicos pretenden valorar económicamente los recursos naturales y buscan la internalización de las externalidades ambientales por el potencial contaminador o por el usuario de un recurso ambiental, algunos de ellos se basan en el principio quien contamina paga, mientras que otros constituyen auténticas ayudas públicas.

En este trabajo presentamos la evolución que se ha dado desde que en el año 1974 se propusiese la aplicación del principio quien contamina paga, por parte de la OCDE, hasta nuestros días y su impacto en el desarrollo de los instrumentos económicos en materia ambiental, haciendo énfasis en aquéllos de más reciente creación, como es el pago por servicios ambientales, el intercambio de deuda por naturaleza o los bonos de conservación. 


\section{1 ¿QUIEN CONTAMINA PAGA O QUIEN CONSUME PAGA?}

Cuando se planteó el principio quien contamina paga fueron muchas las críticas surgidas entorno a su pertinencia, las cuales se basaban en que la exigencia de asumir el coste económico de las externalidades por el potencial contaminador lo que produciría era, a la postre, inflación.

Como ya hemos señalado en la introducción, en el año de 1974, los Estados miembros de la Organización para la Cooperación y el Desarrollo Económico (en adelante OCDE) adoptaron una recomendación conocida como el principio de quien contamina paga (OCDE, 1974).

Dicho principio, nacido en el ámbito de las ciencias económicas, buscaba traspasar el coste de las llamadas externalidades negativas (AYRES \& KNEESE, 1974, p. 74), que en principio soportaría la colectividad en su conjunto, a los agentes potencialmente contaminadores. De este modo nace con un contenido claramente económico y, poco a poco se juridifica, apareciendo desde hace décadas en normas de derecho positivo.

Los costes que se pretenden internalizar, basándose en este principio, son aquéllos provenientes de la prevención de la contaminación, de manera que ella ya no ocurra y comprenden, también, los provenientes del control de la actividad potencialmente contaminante; es decir, se deberán asumir por el contaminador los costos tanto en la etapa de prevención como en la de control.

Como fue originalmente concebido se trataba, entonces, de un "principio fundamental para asignar los costes de las medidas de prevención y control de la contaminación introducidas por las autoridades públicas de los países miembros" (OCDE, 1974).Se planteaba, entonces, como una forma de imputar al contaminador la carga de la lucha contra la contaminación, quien debía asumir el coste de las medidas necesarias para evitarla o reducirla hasta los estándares marcados por dichas autoridades.

Además, se pretendía que los bienes y servicios que causaban contaminación en la producción y / o consumo reflejasen en sus precios el coste de estas medidas ya que las valoraciones económicas pueden y deben ayudar a los agentes económicos en la tarea de tener en cuenta los efectos sobre el medio ambiente cuando toman decisiones de inversión o consumo (GARCÍA, 2001, p. 120 y ss.).

A pesar de lo anterior, hay que tomar en cuenta que existe una estrecha relación entre la política ambiental de un país y su política socioeconómica general (LEFF, 1994, p. 21) y por ello, aunque este principio 
pretende que las autoridades de un país fomenten que sea el contaminador quien asuma el deber de prevenir y controlar la contaminación y sus costes, se puntualiza por parte de la OCDE que, en circunstancias excepcionales, pueden desarrollarse problemas socioeconómicos de tal importancia que justifiquen la consideración de otorgar asistencia gubernamental, cuando:

- Sea necesario aplicar rápidamente un régimen de control de la contaminación especialmente estricto y necesario;

- $\quad$ los objetivos de política ambiental de un país miembro deban realizarse dentro de un tiempo prescrito y específico;

- $\quad$ se otorgue asistencia con el objeto de estimular la experimentación con nuevas tecnologías de control de la contaminación.

Cuando las medidas tomadas para promover los objetivos socioeconómicos específicos de un país tengan el efecto incidental de constituirse en asistencia para efectos de control de la contaminación, el otorgamiento de esa asistencia no sería inconsistente con el principio quien contamina paga, ya que sería una excepción plenamente justificada del principio.

Sin embargo, se recomendaba que, como regla general, los países miembros no apoyasen a los contaminadores en el pago de los costos del control de la contaminación, ya fuese mediante subsidios, ventajas fiscales u otras medidas (OCDE, 1995, p. 5).

Como podemos observar, desde el momento en que se plantea el principio quien contamina paga por parte de la OCDE, se resalta la posibilidad de que existan excepciones que permitan que las autoridades de un determinado Estado puedan apoyar de distintas maneras a los contaminadores, asumiendo ellas, por ende, los costes de la lucha contra la contaminación.Además, se hacía énfasis en que el consumidor era realmente el que pagaba dicha internalización.

De acuerdo con Panayotou (1994), la crítica tradicional al principio quien contamina paga, de quees el consumidor el que debe pagar, no tiene sentido, puesto que realmente el contaminador es el consumidor.

Los contaminadores o los consumidores no sólo deberían pagar el coste de la prevención y el control de la contaminación, sino también el uso de la capacidad asimilativa del medio ambiente.

A partir del año 2002, con la celebración de la Cumbre Mundial de Desarrollo Sostenible, celebrada en Johannesburgo, Sudáfrica, los 
conceptos de consumo responsable, consumo sostenible o responsabilidad social y ambiental de las empresas, se introdujeron con fuerza en el discurso del desarrollo sostenible y la propia declaración final de esta conferencia se refiere a ello, al igual que el Plan de aplicación de las decisiones de la cumbre (PLAN DE APLICACIÓN DE LAS DECISIONES DE JOHANNESBURGO, 2002), en el cual se destaca la necesidad de:

Seguir promoviendo la incorporación de los costos de la protección ambiental y el empleo de instrumentos económicos, sobre la base del criterio de que el que contamina debe, en principio, cargar con los costos de la contaminación, teniendo debidamente en cuenta el interéspúblico y sin distorsionar el comercio ni las inversiones internacionales.

\section{QUIEN CONTAMINA PAGA FRENTE A QUIEN SE BENEFICIA PAGA}

El principio quien contamina paga, además, no es el único principio para la distribución de costes, ya que también tiene sentido el principio "quien se beneficia paga", el cual ha cobrado un enorme protagonismo durante los últimos años. Según éste, aquellos individuos o grupos que reciben o esperan recibir beneficios por actividades de control de la contaminación o acciones de conservación deberían pagar por dicho beneficio.

Un ejemplo claro de lo anterior es el pago por servicios ambientales, del que hablaremos más adelante o los más recientes tratados internacionales en materia climática (ACUERDO DE PARÍS, 2015) o de protección de la biodiversidad, según los cuales el coste de la conservación de la biodiversidad y el control de las emisiones de gases de efecto invernadero (GEI) debe ser soportada, en su mayoría, por los países desarrollados, que son los que más se han beneficiado históricamente por el uso de la atmósfera y, al mismo tiempo, los que se benefician porque estos recursos se encuentren en condiciones sanas.

En definitiva, a medida que los recursos naturales son más escasos o se encuentran sometidos a mayor presión su valor económico sube y, por ende, quienes se beneficien por estos deben "pagar" por ellos. 


\section{CLASIFICACIONES DE LOS INSTRUMENTOS ECONÓMICOS} EN MATERIA AMBIENTAL: LOS INSTRUMENTOS ECONÓMICOS DE ACUERDO CON LOS PRINCIPIOS QUE LOS RIGEN

Son muchas las clasificaciones para los instrumentos económicos. A continuación, exponemos algunas de ellas.

Según Opschoor y Vos (1989), debieran catalogarse de la siguiente manera:

1. Cánones

- Cánones de vertido.

- Cánones por servicio prestado.

- Cánones sobre productos.

- Cánones administrativos.

- Diferenciación a través de impuestos.

2. Ayudas financieras

- Subsidios

- Créditos blandos

- Desgravaciones fiscales.

3. Sistemas de consignación

4. Creación de mercados

- $\quad$ El intercambio de derechos de emisión.

- $\quad$ La intervención del mercado.

- $\quad$ El seguro de responsabilidad.

5. Incentivos financieros para asegurar el cumplimiento.

- Gravámenes de no cumplimiento.

- $\quad$ Depósitos de buen fin. (p. 29-34)

Hay quien distingue entre tasas o cánones, impuestos propiamente dichos, fondos, seguros y sistemas de depósito- reembolso (OCDE,1999).

Dentro de las tasas o cánones estarían:

- cánones por emisión;

- cánones sobre los usuarios; y

- cánones sobre los productos. 
De acuerdo con esta clasificación, los cánones o tasas de emisión son "pagos directos basados en la estimación de la cantidad o la calidad de un contaminante" (OCDE, 1999) y los cánones sobre los usuarios serían:

pagos por el costo de los servicios públicos o colectivos y son esencialmente vistos y usados como un mecanismo financiero por parte de las autoridades locales, por ejemplo: los cánones por la recogida de la basura o por el servicio del agua. En el caso de los recursos naturales, los cánones para los usuarios son pagos por el uso de un recurso natural (Por ejemplo: cuotas de caza, pesca...).

En lo que se refiere a los cánones aplicables sobre productos, estos se imponen sobre productos que generan contaminación, ya sea en su elaboración, consumo o disposición final (por ejemplo: fertilizantes, plaguicidas, baterías...). Estos cánones pretenden modificar los precios finales de estos productos y/o financiar la recolección y/o tratamiento de estos.

También se han clasificado los instrumentos económicos de acuerdo con su propósito:

- aquéllos que buscan incentivar o desincentivar acciones en relación con el medio ambiente;

- $\quad$ los que pretenden financiar acciones o servicios hacia los recursos naturales.

Aunque a veces ambos propósitos pueden convivir, ello puede generar confusiones y que no funcionen tan bien como sería de desear.

Hay quienes, en lugar de hablar de instrumentos económicos, se refieren a instrumentos basados en el mercado (ANDERSEN, 1998), los cuales serían:

- Derechos o cánones;

- Impuestos;

- $\quad$ Sistemas de depósito- reembolso;

- Subsidios; 
- Créditos para la reducción de emisiones;

- Permisos negociables.

El VII Programa comunitario sobre medio ambiente (Diario Oficial de la Unión Europea, serie L 354, 2013) insiste en la necesidad de transformar la economía mundial "en una economía verde e integradora". Para ello propone:

Una combinación adecuada de instrumentos políticos, lo cual permitiría a las empresas y los consumidores comprender mejor cómo repercuten sus actividades sobre el medio ambiente y saber cómo gestionar ese impacto. Entre tales instrumentos políticos cabe citar los siguientes: incentivos económicos, instrumentos de mercado, requisitos en materia de información y herramientas y medidas voluntarias que complementen el marco legislativo y comprometan a las partes interesadas a distintos niveles.

\section{El programa también insiste en la necesidad de un marco que:}

aporte las señales apropiadas a los productores y consumidores a fin de promover la eficiencia en el uso de los recursos y la economía circular. Se adoptarán medidas para seguir mejorando el comportamiento medioambiental de bienes y servicios en el mercado de la Unión a lo largo de todo su ciclo de vida, incluidas medidas para intensificar la oferta de productos ambientalmente sostenibles y propiciar entre los consumidores un cambio significativo a favor de la demanda de esos productos. Esto se conseguirá aplicando una combinación equilibrada de incentivos para los consumidores y las empresas (PYME incluidas), instrumentos de mercado y reglamentaciones para reducir el impacto medioambiental de sus actividades y productos. Los consumidores deben recibir información precisa, fácil de comprender y fiable sobre los productos que adquieren, mediante un etiquetado claro y coherente, incluso en relación con las declaraciones medioambientales. Deben optimizarse los envases para minimizar el impacto medioambiental, y también deben fomentarse los modelos de actividad empresarial eficientes en el uso de recursos, como los sistemas de servicio de productos, incluido el arrendamiento de los mismos (Diario Oficial de la Unión Europea, serie L 354, 2013).

\section{De lo anterior se desprende que en la Unión Europea se distingue} entre:

- Regulaciones;

- Incentivos; 
- $\quad$ Instrumentos de mercado;

- Requisitos de información (etiquetado);

- Instrumentos voluntarios;

De acuerdo con Panayotou (1994), existen 7 categorías de instrumentos económicos, los cuales son:

- $\quad$ Derecho de propiedad;

- Creación de mercados;

- Instrumentos fiscales;

- Tasas;

- Instrumentos financieros;

- Instrumentos de responsabilidad;

- $\quad$ Fianzas de cumplimiento y sistemas de depósito- reembolso.

Según este autor, los derechos de propiedad se basan en el reconocimiento de que la degradación excesiva de los recursos naturales se debe a que las señales de los precios no funcionan para muchos activos ambientales y, por ello, el establecer sobre estos, instrumentos que ayuden a que los precios reflejen la escasez del recurso puede ayudar a tomar mejores decisiones relacionadas con el mismo.

Estos derechos de propiedad, insiste Panayotou, no necesitan ser privados, también pueden ser públicos o comunales, pero necesitan estar bien definidos, ser seguros y transferibles si van realmente a internalizar costes ambientales. Estos derechos son particularmente aplicables a la tierra y suelos, al agua y a los minerales, en forma de derechos sobre el agua, sobre las minas y sobre las tierras, así como a otro tipo de recursos naturales que pudiesen ser fácilmente parcelados o demarcados (PANAYOTOU, 1994, p. 11). 


\section{Los derechos de propiedad pueden ser de tres tipos:}

- Derechos de propiedad propiamente dichos (derechos de agua o derechos sobre la tierra);

- Derechos de uso (licencias, concesiones, certificados de usufructo, derechos de acceso (carreteras, parques...));

- $\quad$ Derecho de desarrollo.

En cuanto a la creación de mercados, esto se refiere a todos los permisos o cuotas transferibles que pueden ser objeto de compraventa. Dentro de estos están:

- Permisos de emisión negociables;

- $\quad$ Cuotas de desarrollo comercializables;

- Cuotas de aguas y de otros recursos negociables (PANAYOTOU, 1994, p. 9).

\section{En lo que se refiere a los instrumentos fiscales, dentro de} estos se distingue entre:

- Gravámenes de contaminación; dentro de estos hay que distinguir entre gravámenes sobre emisiones y gravámenes sobre efluentes;

- Gravámenes sobre productos;

- Impuestos a la exportación;

- Impuestos sobre importaciones;

- Diferenciación fiscal (PANAYOTOU, 1994).

Encuanto a las tasas, dentro de éstas encontramos:

- Tasas de contaminación;

- Tasas sobre los usuarios;

- $\quad$ Tasas de mejoras;

- $\quad$ Tasas de impacto;

- $\quad$ Tasas sobre carreteras;

- Tasas administrativas (PANAYOTOU, 1994). 
En relación a los instrumentos financieros, estos se dividen según Panayotou en:

- Subsidios financieros;

- Créditos blandos;

- Garantías;

- Fondos revolventes;

- Intereses susbsidiados;

- Incentivos para la relocación;

- $\quad$ Fondos sectoriales. (1994)

Por lo que toca a los sistemas de responsabilidad, se incluyen tanto los seguros de responsabilidad como la propia responsabilidad legal y los cargos o multas por incumplimiento, así como los fondos de compensación ambiental.

Por último y en cuanto a los depósitos de buen fin y a los sistemas de depósito- reembolso encontramos:

- $\quad$ Depósitos de buen fin;

- $\quad$ Sistemas de depósito- reembolso;

- $\quad$ Depósitos en caso de accidentes ambientales (PANAYOTOU, 1994).

Otra clasificación de los instrumentos económicos es la que distingue entre (DEMIR, 2011, p.104):

- Instrumentos económicos a nivel macroeconómico. Dentro de estos se incluirían:

- Seguros ambientales;

- Certificados de emisión negociables;

- Fondos;

- Subsidios;

- Deducciones fiscales;

- $\quad$ Fianzas de buen fin;

- Instrumentos económicos a nivel microeconómico, dentro de los cuales se encuadrarían los siguientes: 
- $\quad$ Impuestos y gravámenes;

- Multas y sanciones;

- Acciones de compensación y restauración.

La Ley General del Equilibrio Ecológico y la Protección al Ambiente (en adelante LGEEPA) en México (LGEEPA, 1988), al referirse a los instrumentos económicos en materia ambiental, los define de la siguiente manera:

Se consideran instrumentos económicos los mecanismos normativos y administrativos de carácter fiscal, financiero o de mercado, mediante los cuales las personas asumen los beneficios y costos ambientales que generen sus actividades económicas, incentivándolas a realizar acciones que favorezcan el ambiente.

\section{La LGEEPA distingue en su artículo 22 entre:}

- $\quad$ Instrumentos de mercado;

- Instrumentos fiscales;

- Instrumentos financieros.

\section{Según esta clasificación:}

Son instrumentos de mercado las concesiones, autorizaciones, licencias y permisos que corresponden a volúmenes preestablecidos de emisiones de contaminantes en el aire, agua o suelo, o bien, que establecen los límites de aprovechamiento de recursos naturales, o de construcción en áreas naturales protegidas o en zonas cuya preservación y protección se considere relevante desde el punto de vista ambiental.

En cuanto a los instrumentos económicos de carácter fiscal, de acuerdo al párrafo segundo del artículo 22, son "los estímulos fiscales que incentiven el cumplimiento de los objetivos de la política ambiental. En ningún caso, estos instrumentos se establecerán con fines exclusivamente recaudatorios". 
Por último y en cuanto a los instrumentos financieros la LGEEPA incluye:

los créditos, las fianzas, los seguros de responsabilidad civil, los fondos y los fideicomisos, cuando sus objetivos estén dirigidos a la preservación, protección, restauración o aprovechamiento sustentable de los recursos naturales y el ambiente, así como al financiamiento de programas, proyectos, estudios, investigación científica, desarrollo tecnológico e innovación para la preservación del equilibrio ecológico y protección al ambiente.

La mayoría de los autores se refieren a los instrumentos de mercado como sinónimo de instrumentos económicos; sin embargo, ésta no es la visión de la LGEEPA que, como hemos visto los deja como una categoría diferente a la de los instrumentos fiscales y financieros.

Como hemos podido observar existe un buen número de instrumentos económicos y una gran cantidad de clasificaciones de estos.

Nosotros hemos planteado en trabajos previos la siguiente (GARCÍA, 2017):

1. Instrumentos fiscales

1.1. Gravámenes;

1.2. Ayudas fiscales.

2. Instrumentos financieros

2.1. Fondos;

2.2. Fideicomisos;

2.3. Fianzas;

2.4. Depósitos de buen fin;

2.5. Seguros que cubren los daños ambientales

2.6. Créditos blandos;

2.7. Otros tipos de ayudas públicas (de carácter no fiscal).

3. Instrumentos de mercado

3.1. Sistemas de depósito- reembolso;

3.2. Certificados de emisión negociables;

Un instrumento económico cada vez más utilizado, que puede diseñarse como ayuda pública (subsidio) o como instrumento de mercado 
es el pago por servicios ambientales.

Por otra parte, existe cierta confusión con relación a las multas y sanciones económicas, al igual que con las concesiones, autorizaciones y licencias ambientales.

Hay quienes opinan que todos ellos son instrumentos económicos; sin embargo, nosotros consideramos que no es así.

En el caso de las multas y sanciones económicas en general, son instrumentos correctivos que se ponen en marcha cuando las medidas de prevención y control no han funcionado por algún motivo.

No podemos olvidar que el derecho ambiental tiene un carácter marcademente preventivo. Su finalidad fundamental es prevenir la contaminación y, por ello, la mayoría de sus normas, mecanismos, instrumentos y previsiones responden a este principio.

Es bien sabido que en materia ambiental las acciones correctivas son especialmente ineficaces puesto que una vez que el daño ambiental se ha producido la reparación es difícil, costosa y en ocasiones (como por ejemplo la pérdida de especies) imposible.

Los instrumentos económicos lo que buscan esencialmente es la prevención y el control de la contaminación para que esta no ocura y no se generen, así, las externalidades ambientales.

Además, los instrumentos económicos se basan en la mayoría de las ocasiones en el principio quien contamina paga, que es un principio de marcado carácter preventivo (GARCÍA, 2001).

Desde luego que el derecho ambiental se apoya en una serie de normas que pretenden la reparación del daño en caso de que éste llegue a producirse e incluye, también, multas y sanciones económicas que juegan un papel necesario para desalentar las conductas contaminantes; sin embargo, dichos mecanismos, que entran en funcionamiento a posteriori, es decir, cuando el daño ya se ha producido no cumplen con los requisitos de un instrumento económico, ni comparten su filosofía ni sus reglas de funcionamiento.

En lo que se refiere a las licencias de funcionamiento, concesiones y autorizaciones, señalados en la LGEEPA como instrumentos de mercado, consideramos que tampoco lo son, sino que en estos casos estamos ante instrumentos de regulación directa, requisitos administrativos para llevar a cabo determinada actividad, con una naturaleza jurídica similar a la de las evaluaciones del impacto ambiental, por poner un ejemplo.

Estos instrumentos de regulación directa son condiciones de 
funcionamiento exigidas por el derecho administrativo- ambiental.

Los instrumentos económicos, como ya hemos señalado, son regulaciones indirectas que buscan inducir cambios en el comportamiento de quien realice una conducta con repercusión sobre el medio ambiente.

Siguiendo con lo dispuesto por la LGEEPA consideramos de gran importancia distinguir, al referirnos a los instrumentos fiscales, entre gravámenes, dentro de los cuales estarían tanto los impuestos como los derechos (cánones o tasas) y, por otra parte, las ayudas fiscales, contrarias al principio quien contamina paga y considerada por algunos como “instrumentos fiscales perversos" (UNEP, 2004, p. 22).

En cuanto a los instrumentos financieros, la LGEEPA habla de los seguros de responsabilidad civil por daños al ambiente; sin embargo, nosotros nos referimos, de manera más general a los seguros que cubren los daños ambientales, pues se está generalizando la percepción de la necesidad de utilizar los seguros de una forma mucho más amplia para cubrir hipotéticos daños ambientales.

Hemos incluido, también, dentro de este rubro a los depósitos de buen fin que, aunque son parecidos a las fianzas y en ocasiones comparten con éstas sus bases jurídicas, tienen ciertas peculiaridades y son, asimismo, cada vez más usadas con objetivos ambientales.

En lo que se refiere a los instrumentos de mercado la redacción de la LGEEPA es, como hemos señalado, particularmente desafortunada, por lo que sustituimos los contenidos en ésta por los antes mencionados.

De acuerdo a lo anterior podríamos, también, clasificar los intrumentos económicos de acuerdo a los principios en los cuales se basan. Así, podríamos clasificarlos de la siguiente manera.

- Principios basados en el principio quien contamina paga:

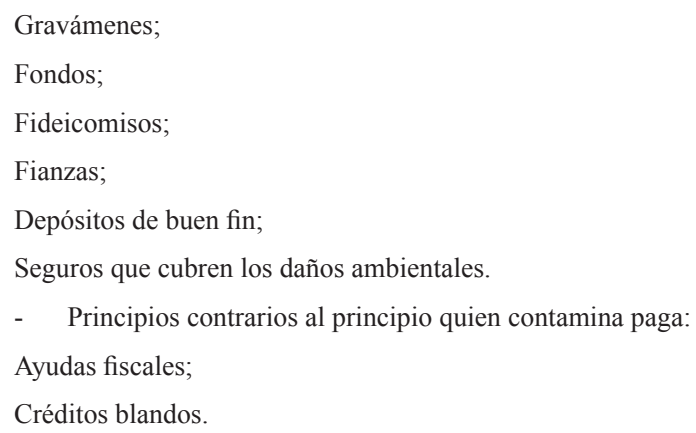




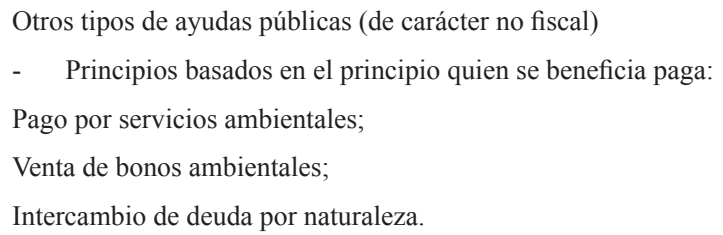

En definitiva, son muchas, como hemos visto, las clasificaciones hechas por la doctrina acerca de los instrumentos económicos en materia ambiental. Nosotros proponemos clasificarlos de acuerdo a los principios de asignación de costes económicos en que se basan.

\section{INSTRUMENTOS ECONÓMICOS BASADOS EN EL PRINCIPIO QUIEN SE BENEFICIA PAGA}

\subsection{Intercambio de deuda por naturaleza}

La Unión Europea en su V Programa de Acción Comunitario en materia ambiental ya proponía la reducción de la deuda externa de países terceros a cambio de que estos aumentasen sus acciones de protección ambiental.

Un ejemplo reciente de este instrumento es el pago, por parte de la Organización no Gubernamental (ONG) The Nature Conservancy, de gran parte de la deuda externa de Seychelles (2016) con la finalidad de que ésta destinase ese dinero a acciones de conservación ambiental.

Esta iniciativa surge de los trabajos y acuerdos del Club de París, en su afán por buscar soluciones sostenibles y coordinadas para los Estados más pobres y con una deuda externa elevada. ${ }^{1}$

En este caso The Nature Conservancy cubrióla deuda de Seychelles a cambio de que este país invirtiese el dinero que tenía destinado a este fin a acciones de conservación ambiental. Para ello se creó el Fondo Seychelles par la Conservación y Adaptación al Cambio Climático y se realizaron una serie de acciones utilizando diferentes instrumentos financieros, como fondos y fideicomisos, los cuales son, también, instrumentos económicos cada vez más utilizados con fines ambientales.

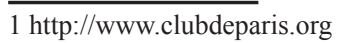




\subsection{Pago por servicios ambientales.}

El pago por servicios ambientales consiste en el otorgamiento de una retribución directa a aquéllos que se ocupan de conservar los ecosistemas que brindan una serie de servicios ambientales necesarios para el bienestar de una comunidad más o menos cercana.

Dicho pago constituye, así, una forma de internalizar, por parte de dicha comunidad, el coste de la prevención de la contaminación y también puede ser visto como una expresión del principio quien se beneficia paga, de acuerdo al cual, el beneficiado por la existencia de determinado hábitat será el que debe pagar por su conservación.

Los programas de pago por servicios ambientales se han planteado hasta el momento de formas muy diferentes, que van desde las ayudas públicas enmarcadas dentro de programas oficiales, hasta mecanismos de pagos voluntarios, realizados a través de fondos.

\subsubsection{El pago por servicios ambientales en México, ¿ayudas públicas o principio quien se beneficia paga?}

El pago por servicios ambientales se ha planteado en México hasta el momento, sobre todo, para fomentar la conservación de áreas naturales como las forestales, acción ésta que permite detener la pérdida de fuentes acuíferas, la desertización y fomenta la captura de carbono, entre otros.

Sin embargo y, a pesar de que hasta el momento el pago por servicios ambientales sólo se ha planteado para la protección de áreas forestales en México, bien podría ser utilizado para la protección de otros ecosistemas.

A pesar de lo anterior, no existe todavía un diseño uniforme en relación a este instrumento; cuestiones como quienes son los beneficiarios de un servicio ambiental, y por ende, quienes deben pagar por dichos servicios o cuanto deben pagar y, por lo tanto cómo debe fijarse su precio, son aspectos que todavía no han sido resueltos.

Tampoco existe claridad en cuanto a qué instrumentos son más efectivos para desarrollar este instrumento ni qué estrategia de política pública adoptar.

El Plan Nacional de Desarrollo para el período 2007-2012 (DOF, 2007) planteaba la creación de dispositivos afines al mecanismo de 
desarrollo limpio (MDL) para el pago por servicios ambientales, aunque estos hoy en día no se han desarrollado. Hasta el momento se han elaborado programas de pago por servicios ambientales, financiados en su mayoría por el sector público, enfocados al agua.

La captura de carbono se consideraba como un servicio ambiental ya en el PND 2007-2012 (DOF, 2007, p. 247) y se planteaba su pago como un instrumento de mercado, el cual no necesariamente tendría que ser financiado por el sector público. De hecho, los instrumentos de mercado se supone que son financiados por los particulares y, por lo tanto, se basan en el principio de "quien contamina paga" o de "quien se beneficia paga", al contrario de lo que sucede con muchos de los esquemas actuales de pago por servicios ambientales, que funcionan como subsidios y, por ende, no están basados en estos principios.

El actual PND 2013-2018 (DOF, 2013) señala en su estrategia 4.4.4., relacionada con la protección del patrimonio natural, entre otros, la necesidad de:

- Promover la generación de recursos y beneficios a través de la conservación, restauración y aprovechamiento del patrimonio natural, con instrumentos económicos, financieros y de política pública innovadores.

- Incrementar la superficie del territorio nacional bajo modalidades de conservación, buenas prácticas productivas y manejo regulado del patrimonio natural.

- Recuperar los ecosistemas y zonas deterioradas para mejorar la calidad del ambiente y la provisión de servicios ambientales de los ecosistemas (2013).

Como mencionamos, ha sido muy habitual en México, al igual que en otros países, cubrir el pago por servicios ambientales a través de ayudas públicas. Así, desde el año 2003, se inició el pago por servicios ambientales en el país a través de cuatro programas:

1. El programa de servicios ambientales hidrológicos (PSAH).

2. El programa para desarrollar el mercado de servicios ambientales por captura de carbono y los derivados de la biodiversidad y para fomentar el establecimiento y mejoramiento de sistemas agroforestales (PSA-CABSA).

3. El proyecto de servicios ambientales del bosque (PSAB).

4. El programa ProArbol.

2 http://www.conafor.gob.mx. 
En la actualidad el Programa Nacional Forestal (PRONAFOR) "apoya a los dueños y poseedores de bosques, selvas, manglares, humedales y zonas áridas, para cuidar, mejorar y aprovechar sustentablemente los recursos forestales presentes en estos ecosistemas". ${ }^{3}$

De acuerdo a sus reglas de operación (DOF, 2014), en su componente de servicios ambientales, el objetivo del programa se centra en:

Otorgar apoyos a personas propietarias o poseedoras de terrenos forestales, que de manera voluntaria deciden participar en el programa de pago por servicios ambientales, con el objeto de incorporar prácticas de buen manejo para promover la conservación y manejo sustentable de los ecosistemas, y fomentar la provisión en el largo plazo de los servicios ambientales, tales como la captación de agua, el mantenimiento de la biodiversidad y la captura y conservación del carbono, los cuales benefician a centros de población o el desarrollo de actividades productivas.

A nivel estatal son muchas las entidades federativas que han iniciado programas de pago por servicios ambientales. Así, la Ley de Desarrollo Forestal Sustentable para el estado de Veracruz (LEY DE DESARROLLO FORESTAL SUSTENTABLE PARA EL ESTADO DE VERACRUZ, 2006) define, en su artículo 2, el objeto de la Ley:

[...] regular y fomentar las acciones de conservación, protección y restauración, producción, ordenación, cultivo, manejo y aprovechamiento que se realicen sobre los ecosistemas forestales, los servicios ambientales que éstos ofrecen, las cuencas hidrológico-forestales y los recursos forestales maderables [...] para propiciar el desarrollo forestal sustentable de la entidad. (Art. 2, 2006)

La ley estatal no hace referencia al papel de los bosques y las selvas en la lucha contra el cambio climático, sin embargo, dedica todo un capítulo a los "servicios ambientales forestales" (LEY DE DESARROLLO FORESTAL SUSTENTABLE PARA EL ESTADO DE VERACRUZ, 2006, capítulo VI), considerados en función de su papel en la regulación del ciclo hídrico.

En este mismo sentido, el Plan Sectorial Forestal 2006-2028 para este mismo estado de Veracruz — el cual fue elaborado con base en 3 http://www.conafor.gob.mx/web/apoyos/pronafor/ 4 http://www.ccmss.org.mx/wp-content/uploads/2014/10/plan_sectorial_forestal_estatalactualizacion_2006_2028.pdf 
el Programa Estratégico Forestal para México 2025 (CONAFOR, 2001) y actualizado en diferentes ocasiones- reconoce que "en relación al tipo de servicio compensado económicamente, destaca el que se refiere a la regulación del régimen hídrico, mientras que el secuestro [captura] de carbono se realiza únicamente en dos unidades de manejo" (PLAN SECTORIAL FORESTAL, 2006, p. 66).

En el Plan se destaca que en la actualidad el pago por servicios ambientales que prestan los ecosistemas forestales cubre 32,533 hectáreas (PLAN SECTORIAL FORESTAL, 2006, p. 42), y, dentro de los programas estratégicos por sector que se establecen en el mismo Plan, el referido a "los servicios ambientales en zonas forestales", incluye ya la captura de carbono. Para ello, señala dentro de sus metas, se pretende " [...] incorporar 285,000 hectáreas del territorio veracruzano, identificadas como 'Tierras Kyoto 3' en esquemas de reducción de emisiones vinculadas al Mecanismo de Desarrollo Limpio" (PLAN SECTORIAL FORESTAL, 2006, p. 66) en un plazo de veinte años.

A nivel municipal destaca, por ser uno de los primeros en el país, el programa FIDECOAGUA del municipio de Coatepec, Veracruz, México.

El Programa se encuentra instituido a través de un fideicomiso público municipal, a través del cual se lleva a cabo la operación y el pago de los servicios ambientales.

El 12 de febrero de 2002 se creó el Fideicomiso para el Pago de Servicios Ambientales (FIDECOAGUA), el 30 de junio del mismo año el Congreso del Estado lo autoriza y el 22 de agosto se publica en la Gaceta Oficial del Estado.

\subsubsection{Otros programas públicos para el pago por servicios} ambientales

A nivel internacional se han desarrollado diferentes programas de pago por servicios ambientales.

En la Unión Europea, dentro de la Política Agrícola Común, "existen desde hace algunos años programas de servicios ambientales que podrían entenderse como una versión de pago por servicios ambientales".

Además, el Reglamento Europeo 1698/2005 (DIARIO OFICIAL DE LA UNIÓN EUROPEA L 277, 2005) "prevé la celebración de "contratos territoriales de explotación", como uno de los instrumentos que 
permiten la compensación de los servicios ambientales que los ganaderos, agricultores y silvicultores generan en el marco de las medidas previstas en este reglamento". (DIARIO OFICIAL DE LA UNIÓN EUROPEA L 277, 2005, p.95)

Estos contratos territoriales son, como señala RodríguezCháves Mimbrero: "instrumentos de apoyo a las políticas de desarrollo rural sostenible, que orientan o incentivan las actividades agrarias hacia la multifuncionalidad y generación de externalidades positivas" (2011, p. 74).

Otras formas de pago por servicios ambientales en España, de acuerdo a esta autora son:

\footnotetext{
- La custodia del territorio

- $\quad$ La propuesta del céntimo forestal.
}

En Estados Unidos, por otra parte, hay una larga historia de pago por servicios ambientales hacia los agricultores (CLAASSEN, 2008, p.737), la cual empezó en los años 30.

La mayoría de los programas de este tipo parten, al igual que sucede en la Unión Europea, de unos contratos entre Administración y administrado, en los que se definen los objetivos a conseguir y las obligaciones de cada uno de ellos.

En la actualidad, el servicio de conservación de recursos naturales, dentro del Departamento de Agricultura tiene distintos programas que buscan, a través de la valoración económica de los diferentes ecosistemas, promover la conservación de estos.

Uno de estos programas es la iniciativa para la restauración de los grandes lagos 5 (GLRI por sus siglas en inglés), cuyo objetivo consiste en apoyar a los granjeros y propietarios de tierras colindantes con estos lagos para que combatan las especies invasoras, protejan las cuencas y líneas de costa y restauren los humedales.

Otro programa de pago por servicios ambientales, también en Estados Unidos, es el Conservation Reserve Program ${ }^{6}$, el cual paga a los granjeros de determinadas zonas por no producir y por llevar a cabo acciones de conservación en sus tierras, con la finalidad de mejorar la calidad del agua, frenar la erosión y mejorar el hábitat de especies en

5 http://www.nrcs.usda.gov/wps/portal/nrcs/site/co/home/

$6 \mathrm{http}: / /$ www.fsa.usda.gov/programs-and-services/conservation-programs/conservation-reserve-program/index 
peligro o amenazadas.

Dentro de los países en desarrollo, Costa Rica ha sido pionero en el uso de este instrumento económico (PAGIOLA, 2008, p.712), desarrollando programas de pago por servicios ambientales relacionados con la provisión de agua y la captura de carbono, entre otros, aunque han sido criticados porque los montos de los pagos son demasiado bajos como para realmente incentivar las conductas deseadas (PAGIOLA, 2008).

En Brasil el programa PROAMBIENTE nació, de acuerdo a de Oliveira: "de la presión de los movimientos populares amazónicos, con el apoyo de instituciones universitarias y de la sociedad civil" (2012, p. 64 y ss.); por su parte, el programa "Bolsa - Floresta" del estado del Amazonas fue creado por la ley 3.135/2007 y contiene 4 diferentes casos de pago por servicios ambientales (OLIVEIRA, 2012). También existen otros dos programas: "Produtor de Águas" y "Mina d'Água", este último en el estado de Sao Paulo.

Todos estos programas se desarrollan a través de pagos directos del gobierno a los propietarios de los recursos naturales, constituyendo, entonces, ayudas públicas.

\subsubsection{Hacia programas de pago por servicios ambientales basados en el principio quien se beneficia paga}

Los instrumentos de mercado parten de la base de que las fuerzas del mercado son muy poderosas. Como señalaba en su momento Adam Smith (1794), el mercado induce a la gente a comportarse por el bien común como si estuvieran guiados por una autoridad superior.

Los instrumentos de mercado constituyen una categoría de instrumentos económicos, normalmente de carácter voluntario, que hacen que resulte conveniente para el potencial contaminador o beneficiario de determinados servicios ambientales el realizar ciertas conductas favorables para el medio ambiente.

Como señalan algunos autores, a pesar de lo anterior, en la actualidad no se puede reconocer un mercado en el que los recursos naturales tengan un precio claro (Smith, 1794).

También es difícil encontrar el nexo de unión entre los titulares de los ecosistemas que prestan servicios ambientales o quienes hacen posible o se encargan de la conservación del recurso natural en cuestión y los directamente beneficiados por esos servicios. 
El pago por servicios ambientales puede orquestarse de muy diferentes formas: en primer lugar, puede ser un pago voluntario $u$ obligatorio, puede recaer en los habitantes del municipio o municipios en los que se encuentre el recurso, en los del estado o estados correspondientes o incluso se podría pensar en relacionarlo con una actividad de las que generan emisiones de gases de efecto invernadero, a nivel nacional o internacional, con la finalidad de equilibrar o compensar dichas emisiones.

Otro aspecto importante a tomar en cuenta es el relativo a la recaudación del pago, que puede realizarse de forma independiente o en el momento en el que la persona que debe pagar realiza alguna otra conducta o cumple con alguna otra obligación.

Asimismo, es importante definir quien se beneficiará del pago, es decir, cual será el destino de los recursos. Lo más lógico sería que el destino fuese el financiamiento de acciones de conservación, vigilancia e incluso restauración de los ecosistemas, aunque también es posible beneficiar a quienes favorecen el buen estado del recurso, aunque no se encuentren geográficamente dentro o alrededor del mismo.

Por último, es necesario definir el procedimiento de gestión de los recursos; una opción sería hacerlo a través de Fondos creados al efecto.

\subsection{Venta de bonos o certificados ambientales}

Cada vez es más habitual encontrarnos con mecanismos financieros que pretenden "vender", ya los servicios ambientales que brinda un ecosistema determinado, como hemos mencionado en el apartado anterior, ya acciones de conservación o de protección ambiental en una zona concreta.

Dichas "ventas" se han organizado a través de mecanismos muy diferentes. Algún ejemplo de lo anterior lo tenemos en calcular, por ejemplo, la huella ecológica de un bien o servicio y cobrar una cantidad extra en base a esos cálculos. También se han orquestado estas "ventas" a través de donativos a fondos concretos para "compensar" el impacto de una actividad determinada sobre el medio ambiente.

Normalmente estos bonos o certificados, que van más allá que los tradicionales certificados de emisión negociables, que surgieron en EEUU y adquirieron cierto auge con el Protocolo de 
Kioto, son de carácter voluntario, pero en algunos países existen, ya, ciertas iniciativas para crear algunos con carácter obligatorio.

\section{CONCLUSIONES}

Primera. Aunque cuando se plantea por vez primera por parte de la OCDE el principio quien contamina paga surgieron numerosas críticas, centradas en que la exigencia de asumir el coste económico de las externalidades por el potencial contaminador lo que produciría sería, a la postre, inflación, hoy esta preocupación ha sido superada puesto que ha sido ampliamente reconocido que el contaminador es el consumidor y que éste debe ser social y ambientalmente responsable.

Segunda. El principio quien contamina paga no es el único principio para la asignación de costes para la prevención y el control de la contaminación ya que también tiene sentido el principio "quien se beneficia paga", según el cual aquéllos que reciben o esperan recibir beneficios por actividades de control de la contaminación o acciones de conservación deberán pagar por dicho beneficio.

Tercera. Parte de la doctrina considera que las multas y sanciones económicas, al igual que las concesiones, autorizaciones y licencias ambientales son instrumentos económicos; sin embargo, nosotros consideramos que no es así.

En el caso de las multas y sanciones económicas, estos son instrumentos correctivos que se ponen en marcha cuando las medidas de prevención y control no han funcionado por algún motivo.

En lo que se refiere a las licencias de funcionamiento, concesiones y autorizaciones, consideramos que tampoco lo son, sino que en estos casos estamos ante instrumentos de regulación directa, requisitos administrativos para llevar a cabo determinada actividad, es decir, condiciones de funcionamiento exigidas por el derecho administrativo- ambiental.

Los instrumentos económicos son regulaciones indirectas que buscan inducir cambios en el comportamiento de quien realice una conducta con repercusión sobre el medio ambiente.

Cuarta. Existen numerosas clasificaciones de los instrumentos económicos en materia ambiental. Nosotros proponemos clasificarlos de acuerdo a los principios económicos en los cuales se basan. Así, tendríamos: 
- $\quad$ Principios basados en el principio quien contamina paga:

Gravámenes;

Fondos;

Fideicomisos;

Fianzas;

Depósitos de buen fin;

Seguros que cubren los daños ambientales.

- $\quad$ Principios contrarios al principio quien contamina paga:

Ayudas fiscales;

Créditos blandos;

Otros tipos de ayudas públicas (de carácter no fiscal).

- $\quad$ Principios basados en el principio quien se beneficia paga:

Pago por servicios ambientales;

Venta de bonos ambientales;

Intercambio de deuda por naturaleza.

\section{BIBLIOGRAFÍA}

ALONSO GARCÍA, E.:El Derecho Ambiental de la Comunidad Europea, Ed. Civitas, Madrid, 1993.

ANDERSEN, M. S.: "Institutional aspecs of market-based instruments", Environmental Policy Research Briefs, núm. 5, European Comission, Dublín, 1998.

AYRES, R. y KNEESE, A.: "Producción, Consumo y Externalidades", Economía del Medio Ambiente, Instituto de Estudios Fiscales, Ed. J. A. Gallego Gredilla, Madrid, 1974, p. 74.

BEAUMONT ROVEDA, E.:Desafios y propuestas para la implementación más efectiva de instrumentos económicos en la gestión ambiental de América Latina y el Caribe: el caso de Argentina, Ed. CEPAL, Argentina, 2004.

CLAASSEN, R., CATTANEO, A. \& JOHANSSON, R.: "Cost-effective design of agri-environmental payment programs: U.S. experience in theory and practice", en, Ecological Economics, n ${ }^{\circ} 65$, 2008, p. 737.

COM 436 final de 21 de septiembre de 2005.

Convención Marco de las Naciones Unidas sobre el Cambio Climático, París, 12 de diciembre de 2015. 
DE OLIVEIRA NUSDEO, A. M.:Pagamento por Serviços Ambientais, Sustentabilidade e disciplina jurídica, Editorial Atlas, Brasil, 2012, p. 64 y ss.

Directrices Comunitarias sobre Ayudas Estatales en favor del Medio Ambiente, Diario Oficial de las Comunidades Europeas C 82 de 1 de abril de 2008.

Economía Verde en el contexto del desarrollo sostenible y erradicación de la pobreza: Una perspectiva desde América Latina y el Caribe, UNEP/ LAC-IG.XVIII/3, VIII Reunión del Foro de Ministros de Medio Ambiente de América Latina y el Caribe Quito, Ecuador, 31 de enero al 3 de febrero de 2012. Recuperado de: http://www.pnuma.org/forodeministros/18-ecuador/ Reunion \%20Expertos/Informe\%20Economia\%20Verde/ESPANOL\%20 Economia\%20Verde\%2016\%20DEC\%202011.pdf

GARCÍA LÓPEZ, T.:Instrumentos económicos en materia ambiental. Bases jurídicas para el diseño de políticas públicas, Ed. Fundación Jacobea, A.C., México, 2017, cap. 3.

GARCÍA LÓPEZ, T.:Quien contamina paga. Principio regulador del Derecho ambiental, Ed. Porrúa, México, 2001, p. 120 y ss.

GARCÍA PACHÓN, M.:Instrumentos económicos y financieros para la gestión ambiental, Ed. Universidad Externado de Colombia, Colombia, 2016.

GARCÍA URETA, A.:Marco Jurídico del procedimiento de impacto ambiental: El contexto comunitario y estatal, Colección Tesis Doctorales Bilbao, Instituto Vasco de Administración Pública, Bilbao, 1994.

HUBER, M.H., RUITENBEEK, H. J. y SERÔA DA MOTTA, R.:MarketBased Instruments for Environmental Policymaking in Latin America and the Caribbean, Ed. The International Bank for Reconstruction, U.S.A., 1998.

JIMÉNEZ HERNÁNDEZ, J.:El tributo como Instrumento de Protección Ambiental, Ed. Comares, Granada, 1998.

LEFF, E.: "Sociología y ambiente: Formación socioeconómica, racionalidad ambiental y transformaciones del conocimiento", en Leff, Enrique, (comp.), Ciencias Sociales y Formación ambiental, Editorial Gedisa, Barcelona, 1994, p. 21.

Ley General del Equilibrio Ecológico y la Protección Ambiental, Diario Oficial de la Federación, 28 de enero de 1988, México. 
Ley de Desarrollo Forestal Sustentable del estado de Veracruz de Ignacio de la Llave, Gaceta Legislativa del Estado de Veracruz, 21 de junio de 2006.

MATHIAS, K. \& VICTURINE, R.: Fondo fiduciario para la conservación. Estudio sobre la inversión para el año calendario 2014, Wildlife Conservation Society, 2015.

Naciones Unidas, El Futuro que queremos, Resolución adoptada por la Asamblea General de las Naciones Unidas, 27 de julio de 2012. http:// www.un.org/es/comun/docs/?symbol=A/RES/66/288

NAUMANN, S. et al:: Design, implementation and cost elements of Green Infrastructure projects. 2011, Final report, cit. en, Institute for European Environmental Policy, Green Infrastructure Implementation and Efficiency, ENV.B.2/SER/2010/0059, Final Report, 2011.

Organization for Economic Co-operation and Development (OECD): Comunicación de la Comisión a los Estados miembros sobre las ayudas estatales para el medio ambiente de 1974, modificada en 1980,1986 y 1994, Paris, 1974, Vid., OCDE, C(74) 223, 21 de noviembre de 1974, 14 ILM, 234, 1975 (V de la 11).

Organization for Economic Co-operation and Development (OECD): Energy Prices and Taxes, International Energy Agency, Francia, 1995, p.5.

Organization for Economic Co-operation and Development (OCDE), Economic Instruments for Pollution Control and Natural Resources Management in OECD Countries: a survey, 1999.

OPSCHOOR, J.B. y VOS, H.B.:Instruments Economiques pour la Protection de l'Environnement, OCDE, París, 1989, cit. en La Fiscalidad y el Medio Ambiente, op. cit., pp. 29-34.

PANAYOTOU, T.:Economic Instruments for Environmental Management and Sustainable Development, United Environment Programme, 1994.

PARK, C. y ALLABY, M.:A Dictionary of Environment and Conservation, 2a. Ed., Oxford University Press, 2013.

Plan de Aplicación de las Decisiones de Johannesburgo, Diario Oficial de las Comunidades Europeas, serie L 242 de 10 de septiembre de 2002.

Plan Sectorial Forestal 2006-2028 para el estado de Veracruz, Acervo del Consejo Civil Mexicano de septiembre de 2006

Plan Nacional de Desarrollo 2007-2012, Diario Oficial de la Federación de 
31 de mayo de 2007.

Plan Nacional de Desarrollo 2013-2018, Diario Oficial de la Federación de 20 de mayo de 2013.

I Programa de acción comunitario en materia ambiental. DOCE C 112 de 20 de diciembre de 1973.

II Programa de acción comunitario en materia ambiental. DOCE C 139 de 13 de junio de 1977.

III Programa de acción comunitario en materia ambiental, DOCE C 46 de 17 de febrero de 1983.

IV Programa de acción comunitario en materia ambiental, DOCE C 328 de 7 de diciembre de 1987.

V Programa de acción comunitario en materia ambiental, DOCE C 138 de 17 de mayo de 1993.

VII Programa comunitario sobre medio ambiente, Diario Oficial de la Unión Europea L/354/171, 28 de diciembre de 2013.

Programa de las Naciones Unidas para el Medio Ambiente, Hacia una economía verde. Guía para el desarrollo sostenible y la erradicación de la pobreza. Sintesis para los encargados de la formulación de políticas, Francia, PNUMA, 2011. http://www.unep.org/greeneconomy/Portals/88/ documents/ger/GER_synthesis_sp.pdf

Programa General de Acción de la Unión en materia de Medio ambiente hasta 2020, "Vivir bien, respetando los límites de nuestro planeta", del Parlamento Europeo y del Consejo, 20 de noviembre de 2013.

Programa Nacional Forestal, Diario Oficial de la Federación de 20 de diciembre de 2014.

Reglamento (CEE) n 2078/92 del Consejo, de 30 de junio de 1993.

RODRÍGUEZ-CHÁVES MIMBRERO, B.: "El contrato territorial y el contrato territorial de zona rural: dos instrumentos para promover una gestión sostenible del medio rural". Ambienta, $\mathrm{N}^{\mathrm{o}}$ 97, 2011, p. 66-82.

ROLFE, C. y NOWLAN L.:Economic Instruments And The Environment: Selected Legal Issues, Ed. Ann Hillyer Barrister \& Solicitor, Canada, 1993.

SARUKHÁN et. al.;Capital Natural de México, Acciones Estratégicas para su Valoración, Preservación y Recuperación, México, Comisión Nacional para el Conocimiento y Uso de la Biodiversidad, 2012. 
Secretaría de Desarrollo Social (SEDESOL):Los instrumentos económicos aplicados al medio ambiente, México, 1992.

SMITH, A.:Investigación de la naturaleza y causas de la riqueza de las Naciones, 1776, versión española, Editora Oficina de Viuda e Hijos de Santander, Valladolid, 1794.

SUÁREZ, Antonio et. al.:Infraestructura verde y corredores ecológicos de los pedregales: ecología urbana del sur de la ciudad de México, México, Universidad Nacional Autónoma de México, 2011.

Tratado de la Unión Europea, 1992.

United Nations Environment Programme (UNEP): The Use of Economic Instruments in Environmental Policy: Opportunities and Challenges, 2004.

Artigo recebido em: 23/03/2018.

Artigo aceito em: 07/05/2018.

\section{Como citar este artigo (ABNT):}

LÓPEZ, T. G. DEL PRINCIPIO QUIEN CONTAMINA PAGA AL PRINCIPIO QUIEN SE BENEFICIA PAGA... Veredas do Direito, Belo Horizonte, v. 15, n. 31, p. 37-66, jan./abr. 2018. Disponível em: <http:// www.domhelder.edu.br/revista/index.php/veredas/article/view/1262>. Acesso em: dia mês. ano. 